
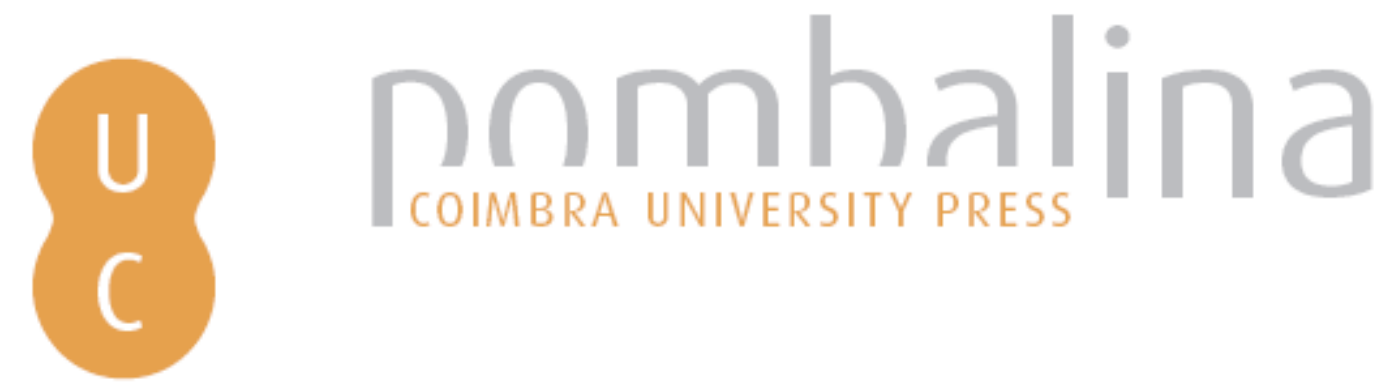

\title{
Reflexivität, Perspektivität und Normativität: systemtheoretische oder transzendentalpragmatische Selbstbezüglichkeit?
}

\author{
Autor(es): $\quad$ Landenne, Quentin \\ Publicado por: Imprensa da Universidade de Coimbra \\ URL \\ persistente: URI:http://hdl.handle.net/10316.2/31634 \\ DOI: DOI:http://dx.doi.org/10.14195/978-989-26-0205-9_12 \\ Accessed : $\quad$ 26-Apr-2023 07:38:11
}

A navegação consulta e descarregamento dos títulos inseridos nas Bibliotecas Digitais UC Digitalis, UC Pombalina e UC Impactum, pressupõem a aceitação plena e sem reservas dos Termos e Condições de Uso destas Bibliotecas Digitais, disponíveis em https://digitalis.uc.pt/pt-pt/termos.

Conforme exposto nos referidos Termos e Condições de Uso, o descarregamento de títulos de acesso restrito requer uma licença válida de autorização devendo o utilizador aceder ao(s) documento(s) a partir de um endereço de IP da instituição detentora da supramencionada licença.

Ao utilizador é apenas permitido o descarregamento para uso pessoal, pelo que o emprego do(s) título(s) descarregado(s) para outro fim, designadamente comercial, carece de autorização do respetivo autor ou editor da obra.

Na medida em que todas as obras da UC Digitalis se encontram protegidas pelo Código do Direito de Autor e Direitos Conexos e demais legislação aplicável, toda a cópia, parcial ou total, deste documento, nos casos em que é legalmente admitida, deverá conter ou fazer-se acompanhar por este aviso.

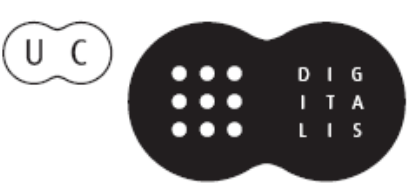


Edmundo Balsemão Pires

Burkhard Nonnenmacher

Stefan Büttner-von Stülpnagel

Editors

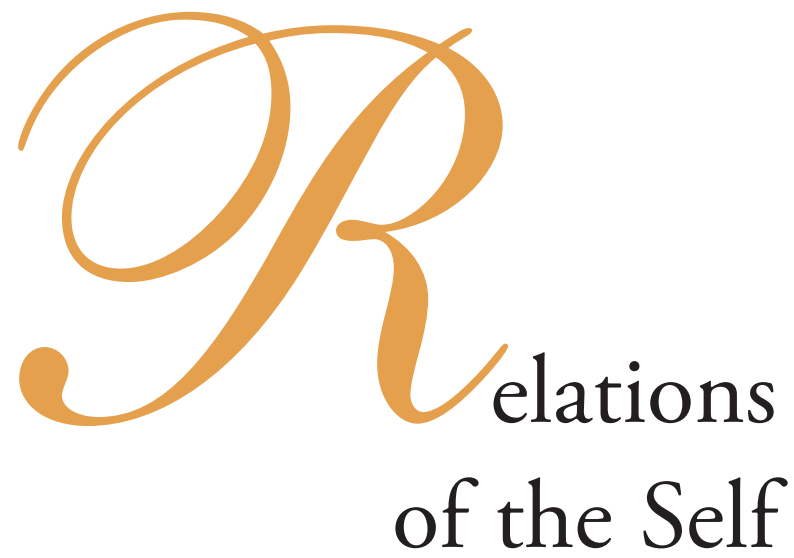




\section{Quentin Landenne}

Bruxelles

\section{REFLEXIVITÄT, PERSPEKTIVITÄT UND NORMATIVITÄT. SYSTEMTHEORETISCHE ODER TRANSZENDENTALPRAGMATISCHE SELBSTBEZÜGLICHKEIT?}

Das Ziel meines Vortrags ist es, die von Niklas Luhmanns Systemtheorie und von Karl Otto Apels transzendentaler Pragmatik entwickelten theoretischen Fassungen der Reflexivität und der Selbstbezüglichkeit zu diskutieren, indem ich die systematische Artikulation zwischen den Begriffen der Reflexivität, der Perspektivität und der Normativität innerhalb beider Theorien besonders hinterfrage. Um diese Artikulation zu erläutern, werde ich zuerst die Art und Weise darstellen, wie beide Autoren eine Moraltheorie bilden, d.h. wie sie den moralischen Standpunkt bestimmen, besonders dem meta-moralischen Standpunkt der Theorie der Moral selbst gegenüber. Luhmann und Apel haben diesbezüglich antithetische Positionen. Die Frage: „Ist eine Theorie der Moral moralfrei?“ führt dann zum allgemeiner epistemologischen Problem des reflexiven Verhältnisses zwischen einer Theorie und ihrem Gegenstandsbereich.

Bestimmte Gegenstandsbereiche, wie z.B. Geschichte, Gesellschaft oder Erkenntnis, können, ihrem wesentlich reflexiven Status nach, die Theorie davon in sich einbeziehen, indem diese Bereiche die notwendigen Bedingungen der Sinnkonstitution einer Theorie überhaupt bilden. Eine umfassende Sozialtheorie muss sich ja als sozialer Gegenstand reflektieren, ohne dabei ihren spezifischen Charakter als Theorie zu verlieren. Die empirische Theorie muss sich darüber hinaus auf eine Theorie der Reflexivität stützen, um die wechselseitige Kongruenz zwischen ihren beiden Polen, nämlich der Theorie und ihrem Gegenstandsbereich, zu sichern.

Die hier verteidigte Hypothese lautet: wenn zwei sozial eingerichtete Theorien der Reflexivität sich in einer epistemologischen Opposition entgegensetzen, wird der theoretische Vorrang nur von derjenigen Theorie behauptet, die sich selbst als Standpunkt innerhalb ihres Gegenstandsbereiches relativieren kann, ohne dabei sich selbst als vorrangigem Standpunkt bzw. als theoretischer Metaperspektive zu widersprechen. Dieser Hypothese nach muss sich die Auseinandersetzung mit den sozialen Theorien Luhmanns und Apels in der Synthese der Reflexivität und der Perspektivität entscheiden. Die These meiner Argumentation lautet, dass eine solche theoretische Synthese nur mittels der Kategorie der Normativität konsistenterweise möglich ist. 


\section{1) Kann eine Theorie der Moral eine moralfreie Theorie sein?}

\section{a) Luhmann: Die Reduktion der Moral auf ihre systemische Funktion}

In verschiedenen Aufsätzen, die 2008 unter dem Titel Die Moral der Gesellschaft in einem posthumen Band veröffentlich wurden, vertritt Luhmann die These einer wertfreien bzw. moralfreien Theorie der Moral. Er widerstreitet dem Argument, dass die Einsicht des Gültigkeitsgrunds von Normen oder Werten notwendigerweise eine Anerkennung dieser Gültigkeit voraussetzt, und zwar eine - positive oder negative - Stellungnahme demgegenüber impliziert. Das Erkenntnisinteresse des Systemtheoretikers geht im Gegenteil stets auf „die Faktizität der Moral und auf ihre Stellung im Variablengefüge gesellschaftlicher Systeme; wie der Moralist die Moral sich vorstelle, das sei nur ein Moment dieser Faktizität“ "

Um einen von der Verschmelzung zwischen Normativität und Faktizität nicht infizierten Zugang zum Gegenstand „Moral“ zu ermöglichen, begreift Luhmann die Ethik als „Reflexionstheorie der Moral“, d.h. als Selbstbeobachtung und Selbstbeschreibung der Funktion des Moralcodes, indem er sich von anderen sozialen Codes (wie Wahrheit, Gerechtigkeit oder Macht) ausdifferenziert. Diese Ethik liefert der Moral eine Moraltheorie, in welcher sie ihre eigenen Probleme reflektieren kann². Die Moral in diesem Sinne kommt nicht wesentlich von einem normativen Sollensbegriff oder von kontrafaktischen Ansprüchen her. Moral ist eine Art systemische Kommunikation, die soziale Komplexität reduziert, indem sie das Problem der doppelten Kontingenz beantwortet. Das Problem der doppelten Kontingenz bedeutet, dass es unmöglich ist für zwei soziale Wesen, die sich als Ego und Alter voneinander differenzieren, das Verhalten des Anderen adäquat zu antizipieren. Ego kann das Verhalten von Alter nur mittels seines eigenen Interpretationscodes, also mittels einer für Alter fremden Selektionsweise deuten und vorsehen. Da Alter die fremde Umwelt von Ego bewohnt, erscheint das Verhalten von Alter als zufällig für Ego, als anders sein könnend. Dieses Kontingenzverhältnis ist verdoppelt, weil es von Ego und von Alter als wechselseitig erfasst wird ${ }^{3}$. Der Moralcode, der als Stabilisierung und Normalisierung der Erwartungen funktioniert, antwortet auf das Problem der doppelten Kontingenz zwischen Personen. Die Perspektiven von Ego und Alter sind durch die Selbstidentifikation des Egos zu seinem Alter als Alter Ego und durch ihre Kommunikation unter der Bedingung der wechselseitigen Achtung integriert ${ }^{4}$. Die spezifische Funktion des Moralcodes moderner Gesellschaften beruht denn auf der Differenzierung Achtung/Missachtung. Die systemtheoretische Perspektive behauptet also nicht nur, moralfrei und normativ neutral zu sein, sondern sie reduziert darüber hinaus die Normativität des moralischen Standpunkts und des Gegensatzes Achtung/Missachtung auf ihre gesellschaftliche Funktion als Komplexitäts- und Kontingenzreduktion.

\footnotetext{
${ }^{1}$ Niklas Luhmann, Die Moral der Gesellschaft, Frankfurt 2008, S.56, 57.

${ }^{2}$ Ibid., S.271, 281.

${ }^{3}$ Niklas Luhmann, Soziale Systeme, Frankfurt 1987, S.148 ff.

${ }^{4}$ Ibid., S.100-102, 110.
} 


\section{b) Transzendentale Pragmatik: moralische Normativität und Performativität}

Der von Luhmann vorgenommenen strikten Trennung zwischen kognitiven Ansprüchen auf Wahrheit und praktischen Ansprüchen auf Normativität ist von J. Habermas und K.-O. Apel in ihrem Programm einer Theorie des kommunikativen Handelns und einer Diskursethik stark widersprochen worden. Die Diskursethik vertritt das Prinzip eines moralischen Kognitivismus, der die normative Richtigkeit als einen zur Wahrheit analogen Gültigkeitsanspruch betrachtet ${ }^{5}$. Die Normativität moralischer Normen ist hier zuerst von innen eingesehen, statt auf ihre soziale Funktion reduziert und objektiviert zu werden. Sie ist durch die konstitutive Normativität des Sprachhandelns rational begründet, d.h. sie kommt aus der Performativität der Sprache, kraft derer der Sprecher tut, was er sagt, gerade indem er es sagt. Diese Performativität erfordert eine Kongruenz des Tuns und des Sagens und der Sprecher verpflichtet sich seinen Gesprächspartnern gegenüber dazu, diese Kongruenz praktisch zu respektieren. Die Reflexion auf die konstitutiven Voraussetzungen des Diskurses offenbart eine Reihe kontrafaktischer Antizipationen und Gültigkeitsansprüche (auf Wahrheit, normative Richtigkeit, Wahrhaftigkeit), zu denen jeder Diskursteilnehmer sich ipso facto verpflichtet, da andernfalls der Diskurs sich in performativem Widerspruch zerstören würde. Auf der Basis dieser Sprachpragmatik und diesen kontrafaktischen universalen Voraussetzungen der Argumentation als reflexive Form des kommunikativen Handelns überhaupt ${ }^{6}$ erläutert die Diskursethik den moralischen Standpunkt. Sie erklärt den Sinn dieses moralischen Standpunktes mittels einer diskursiv-reflexiven Rekonstruktion der psychosozialen Dezentra-tionskompetenz bzw. „ideal role-taking“ und mittels der intersubjektiv prozeduralen Transformation des Universalisierungstests des Kantischen kategorischen Imperativs. Der moralische Standpunkt wird in ein intersubjektives Verfahren integriert, welches die Gültigkeit einer Norm überprüft, insofern diese bei allen Betroffenen eine allgemeine, vernünftig begründete und deshalb zwangslose Zustimmung gewinnen kann ${ }^{7}$.

Auf der gemeinsamen Basis der Diskursethik unternimmt die von Apel entwickelte transzendentale Pragmatik eine reflexive Letztbegründung der praktischen Vernunft und, darüber hinaus, der Moral. Die nichthintergehbaren Voraussetzungen aller realen Diskurse und die kontrafaktische Antizipation einer idealen Kommunikationsgemeinschaft fungieren wie ein regulatives Ideal für die realen Kommunikationsgemeinschaften. Jedes Bestreiten der Notwendigkeit dieser Letztbegründung, sei es beim kritischen Rationalismus, dem Skeptizismus oder dem Relativismus, fällt in einen performativen Widerspruch, weil es pragmatisch voraussetzen muss, was es inhaltlich leugnet. Eine Moraltheorie kann für Apel normativ nicht neutral sein, weil die pragmatischen Voraussetzungen ihrer Argumentation die regulativen Prinzipien einer intersubjektiven Moral bilden, auch wenn die Moraltheorie selbst keine positive bzw. präskriptive Moral ist.

Die Bestimmung des normativen Sinnes eines moralischen Standpunkts in der Diskursethik steht im Gegensatz mit dem Moralbegriff der Systemtheorie. Die von Luhmann vorgenommene

\footnotetext{
${ }^{5}$ Jürgen Habermas , Erläuterung zur Diskursethik, Frankfurt 1991, S.11.

${ }^{6}$ Ibid., S.61.

${ }^{7}$ Ibid., S.53, 54 .
} 
Reduktion der Moral auf ihre systemische Funktion relativiert die moralische Normativität zugunsten ihrer Faktizität und begreift die erste als zufällig, da sie durch funktionale Äquivalente (wie Recht oder Liebe) ersetzbar ist ${ }^{8}$. Diese Reduktion ist nicht auf den empirischen Bereich der soziologischen Beschreibung begrenzt, sondern sie beansprucht eine viel weitere epistemologische Tragweite und betrifft die Grundlage der Sozialtheorie selbst. Luhmann betrachtet allerdings die rationale Begründung einer allgemeinen Moral nicht nur als ein unnützliches Unternehmen, sondern auch als kontraproduktiv und paradox, da es die Moral fraglich macht, gerade indem es ihr Begründungsbedürfnis explizit zugibt. Was hier geleugnet wird, ist die Möglichkeit eines außergesellschaftlichen Standpunktes, der auf dem transzendentalen Begriff der Einen Vernunft für die Eine Moral beruhen würde. Für Luhmann gilt: „vielleicht geht es ohne Vernunft besser“; er reduziert ja den Vernunftbegriff auf seine schlichte anthropologische Funktion?.

Mit dieser funktionalistischen Reduktion der moralischen Normativität und der damit verbundenen naturalistischen Reduktion der Vernunft fällt die luhmannsche Theorie, so Apel, in einen performativen Widerspruch mit den von der Theorie selbst, als diskursivem und argumentativem Handeln, implizierten kontrafaktischen Voraussetzungen. Der Soziologe kann zwar die moralische Normativität seines Gegenstandsbereichs methodologisch einklammern und seine empirischen Analysen neutralisieren; aber eine Theorie, die die Universalität und die Notwendigkeit der Gültigkeitsansprüche auf Wahrheit oder normative Richtigkeit leugnet, widerspricht ihren eigenen pragmatischen Voraussetzungen.

Wie Luhmann betont, erscheinen die internen und externen Perspektiven auf die Moral als antithetisch und unvereinbar ${ }^{10}$; die eine kann $\mathrm{zu}$ der anderen artikuliert werden, nur insofern sie die andere als Derivat ihrer selbst reduziert. Systemtheorie und Transzendentalpragmatik können sich nicht als subkonträr miteinander arrangieren, als ob sie gleichzeitig aber auf verschiedenen epistemologischen Niveaus gültig sein können; sie sind in einem Kampf um den theoretischen Vorrang oder Primat engagiert, der weit über das spezifische Problem der Bestimmung des moralischen Standpunktes geht; dies erfordert ihre jeweilige Theorie der Reflexivität auseinanderzusetzen.

\section{2) Reflexivität und Theorie der Perspektive : Luhmann versus Apel}

In einem Kampf um den theoretischen Primat, so Luhmann, muss eine totalisierende Theorie die gegnerische theoretische Perspektive reduzieren und ihr einen abgeleiteten Status und eine objektivierende Explikation geben ${ }^{11}$. Aber sie muss sich auch vor der Reduktionsstrategie des Gegners hüten, indem sie von sich selbst eine objektivierende Erklärung gibt, die reflexiv genug sein soll, um äußere Objektivierungen unnützlich zu machen. Paradoxerweise kann sich eine totalisierende Theorie gegen fremde Totalisierung

\footnotetext{
${ }^{8}$ Niklas Luhmann, Die Moral der Gesellschaft, Frankfurt 2008, S.101, 135.

${ }^{9}$ Niklas Luhmann, Die Moral der Gesellschaft, Frankfurt 2008, S.272, 281-283.

${ }^{10}$ Niklas Luhmann, Die Moral der Gesellschaft, Frankfurt 2008, S.282.

${ }^{11}$ Niklas Luhmann, Die Moral der Gesellschaft, Frankfurt 2008, S.68 ff.
} 
nur immunisieren, indem sie sich in ihren eigenen Gegenstandsbereich reflexiv einbezieht, d.h. indem sie sich selbst als Theorie relativiert ${ }^{12}$. Die besondere Frage der moralischen bzw. normativen Implikationen einer Moraltheorie geht jetzt zum allgemeinen Problem des reflexiven Verhältnis einer Theorie mit ihrem Gegenstandsbereich über.

Setzen wir uns jetzt mit den von der Systemtheorie und der Transzendentalpragmatik entwickelten verschiedenen Begriffen der Reflexivität und der Selbstbezüglichkeit auseinander! Ihr gemeiner Ausgangspunkt liegt in dem Bruch mit der traditionellen Subjekt-Objekt Spaltung der Bewusstseins-philosophie ${ }^{13}$. Beide Theorien gehen von einer Subjekt-ObjektEinheit aus, sei es das selbstreferentielle System oder die diskursive Reflexivität. Aber sie begreifen diese Subjekt-Objektivität durch zwei diametral entgegengesetzte Reflexivitätsmodelle.

Die Reflexivität der Systemtheorie ist eine objektive Subjekt-Objektivität: die Sozialtheorie muss sich ausgehend von ihrem Objekt selbst reflektieren. Sie ist die Autologie des sozialen Systems selbst. Luhmann vertritt zwar einen radikalen Konstruktivismus: verschiedene Begriffe bilden verschiedene Welten und die Beschreibung vollzieht das Beschriebene ${ }^{14}$. Aber dieser Konstruktivismus setzt eigentlich einen fundamentalen Naturalismus voraus, wonach selbstreferentielle Systeme im Allgemeinen und Gesellschaft als einziges Objekt sozialer Theorie im Besonderen das reale und quasi-biologische Subjekt ist, das diese Theorie erzeugt. Die soziologische Beschreibung ist nur ein relatives Moment der Autopoiesis, kraft derer das System sich identifiziert und sich von seiner Umwelt ausdifferenziert ${ }^{15}$. Nur retrospektiv kann sich die Systemreflexion als ein Ganzes selbst beschreiben; sie kommt immer zu spät, um die von ihren eigenen Operationen vergrößerte Weltkomplexität ganz zu fassen $^{16}$. Die Finalität bzw. die Funktion der Systemreflexion beschränkt sich dann auf die Komplexitätsreduktion der Umwelt eines ausdifferenzierten Subsystems, ist also ein Selbsterhaltungsmittel dieses Subsystems.

Apels Diskurstheorie begründet ihr Reflexivitätsmodell auf einer subjektiven bzw. intersubjektive Subjekt-Objektivität: Reflexivität ist damit als ein wechselseitiges Subjekt-cosubjekt-verhältuis zu verstehen, das eine apriorische Bedingung der Argumentation um damit des als defizitäre Argumentationsweise begriffenen Denkes darstellt ${ }^{17}$. Die transzendentale Argumentation rekonstruiert den subjektiven und objektiven Pol der Selbstreflexion performativer Sprechakte, nämlich das Sagen und das Gesagte, um zu bestimmen, wie das Gesagte mit dem Sagen übereinstimmen soll. Apel begreift ja die Performativität als ein transzendentalpragmatisch transformiertes Analogon des spekulativen Modells der Tathandlung Fichtes. Die reflexive Identität des Sagens und des Gesagten, als konstitutives Prinzip der Argumentation, ist zugleich als regulatives Prinzip und als Kohärenzpflicht für jeden

\footnotetext{
${ }^{12}$ Ibid., S.59, 60; Niklas Luhmann, Soziale Systeme, Frankfurt 1987, S.653.

${ }^{13}$ Niklas Luhmann, Soziale Systeme, Frankfurt 1987, S.595.

${ }^{14}$ Niklas Luhmann, Die Moral der Gesellschaft, Frankfurt 2008, S.270; Niklas Luhmann, Die Gesellschaft der Gesellschaft, Frankfurt 1998, S.16, 33, 1128 ff.

${ }^{15}$ Niklas Luhmann, Die Gesellschaft der Gesellschaft, Frankfurt 1998, S.22, 34, 69.

${ }^{16}$ Niklas Luhmann, Soziale Systeme, Frankfurt 1987, S.602.

${ }^{17}$ Karl-Otto Apel, "Types of rationality To-Day: the continuum of Reason between Science and Ethics ", in T.Geraets (ed.), Rationality To-Day, Ottawa 1979, p.307-350; p.329, 332.
} 
Teilnehmer realer Diskussionen projiziert. Dieser Kohärenzpflicht nach erscheint die diskursive Reflexivität performativer Akte als eine wesentliche Normativitätsquelle. Die ideale Kommunikationsgemeinschaft ist ein moralisch regulatives Sollen für jede reale Kommunikations-gemeinschaft; dieses Sollen ist die kontrafaktische Projektion des transzendental konstitutiven Sollens der Argumentation oder der diskursiven Reflexion. Die transzendentale Reflexion geht vom Gesagten zum Sagen, von der Tatsache zur Tathandlung bzw. zum Tun der Vernunft, ein Tun das sich im empirischen Bewusstsein durch das praktisches Bild des Sollens offenbart. Apels Diskursethik leistet eine transzendentale Rekonstruktion des moralischen Standpunktes: das konstitutive Sollen des transzendentalen Standpunktes wird daher ein regulatives und moralisches Sollen für jeden empirischen Diskursteilnehmer. Da der performative Widerspruch eine bestimmte Selbstentfremdung des Diskurses ist, ist die Finalität der transzendental-pragmatischen Reflexivität die Emanzipation als Selbstbefreiung des praktischen und normativen Potentiellen des kommunikativen Handelns.

Auf der Basis dieser Reflexivitätsmodelle entwickeln sowohl die Systemtheorie als auch die Transzendentalpragmatik eine Theorie der Perspektiven, die es ermöglicht, nicht nur die Komplexität und die Pluralität ihrer Gegenstandsbereiche zu rekonstruieren, sondern auch sich selbst als theoretische Perspektive in diesen Bereichen reflexiv einzubeziehen.

Luhmanns Theorie bildet einen Perspektivismus der selbstreferentiellen Systeme, nach dem die Einheit der verschiedenen sozialen Systeme nur innerhalb jedes ausdifferenzierten Subsystems zu fassen ist ${ }^{18}$. Indem sie ihre Gegenstände als differenzierte Systeme begreift, kann die Theorie auch ihre eigene differenzierte Selbstbezüglichkeit und darüber hinaus ihre perspektivische Relativität annehmen. Eine systemtheoretische soziologische Untersuchung „kann selbst nichts anderes sein als ein selbstreferentielles Sozialsystem, und zwar eines unter vielen, ein Subsystem eines Subsystems eines Subsystems der Gesellschaft "19. Eine Reflexionstheorie ist nur ein Moment der Autopoiesis, kraft derer das Subsystem sich erzeugt, indem es sich in seiner von seiner Umwelt differenzierten Identität reflektiert. Der Primat des autopoietischen Prozesses vor der theoretischen Reflexion führt mit sich den Verzicht des Apriorismus, die Ablehnung aller Absolutheit und aller Äußerlichkeit, Hierarchie oder Asymmetrie in der Beziehung zwischen Erkenntnis und Objekt ${ }^{20}$. Die Systemtheorie, als Performanz des wissenschaftlichen Subsystems, relativiert sich ganz als Teil ihres Gegenstandsbereichs, wird nur eine Perspektive unter anderen, ohne ontologischen Primat oder epistemologischen Vorrang vor anderen subsystemischen Perspektiven.

Apels Transzendentalpragmatik entwickelt ihrerseits ihre Theorie der Perspektiven als Selbstdifferenzierung der praktischen Vernunft in verschiedene Rationalitätstypen bzw. Erkenntnisinteressen. Der Begriff „Erkenntnisinteresse“ wurde von Habermas auf einer Kantisch-Fichteschen Basis eingeführt. Er bezeichnet die Grundorientierungen, die den Forschungsprozess der empirischen Wissenschaften einrichten, den „quasi-transzendentalen“ Rahmen ihres Zugangs zur Wirklichkeit bilden und den Sinn ihrer Erkenntnisweisen und

\footnotetext{
${ }^{18}$ Niklas Luhmann, Soziale Systeme, Frankfurt 1987, S.654.

${ }^{19}$ Ibid., S.660.

${ }^{20}$ Ibid., S.647, 654, 656.
} 
Methoden bestimmen. Die empirisch-analytischen Wissenschaften, besonders die Naturwissenschaften, die nomologische Erklärungen benutzen, haben ein instrumentales Erkenntnisinteresse an der technischen Manipulierung der Wirklichkeit. Hermeneutische bzw. Geistes- oder Humanwissenschaften verfolgen ein Interesse an Sinnverständigung und praktisch orientiertem wechselseitigen Verständnis. Ein drittes Erkenntnisinteresse ist Grund der Reflexion auf die spezifische Verbindung zwischen Erkenntnis und Interesse, auf die Identität der Vernunft und des Vernunftwillens; es ist ein Interesse zur Emanzipation, weil der Vollzug zur Reflexion sich als Emanzipationsbewegung weiß ${ }^{21}$. Dieses dritte Interesse charakterisiert unter anderen die Psychoanalyse, aber auch die kritische Sozialtheorie, in welcher die Diskursethik von Habermas und Apel anzusiedeln sind. Aus diesem emanzipatorischen Interesse sollen die Menschen als Objekte der Humanwissenschaften auch zugleich die Subjekte dieser Selbstreflexion werden ${ }^{22}$.

Die drei Erkenntnisinteressen sind von Apel transzendental rekonstruiert als Standpunkte einer Selbstdifferenzierung der praktischen Vernunft, als füreinander komplementäre und sich vermittelnde Momente derselben vernünftigen Aufgabe. Diese Rationalitätstypen sind also kognitiv autonome, transzendental rekonstruierte, normativ differenzierte und teleologisch orientierte Stanpunkte.

\section{Schluss}

Der Kampf um den Vorrang der reflexionstheoretischen Perspektiven spielt sich dann wohl in Bezug auf die Kategorie der Normativität ab. Luhmanns Systemtheorie, blind für die Performativität der Sprache, löst alle normative Differenzierung zwischen dem Sein und dem Sollen auf und zerstört insofern die Grundlage einer möglichen und konsistenten Unterscheidung zwischen Ideologie und Ideologiekritik. Diese Ablehnung aller normativen Differenzierung kommt grundsätzlich von einer Naturalisierung der theoretischen Reflexivität her: indem sie sich völlig in ihrem Gegendstandsbereich relativiert, beseitigt die Theorie alle Asymmetrie zwischen dem empirischen und dem meta-empirischen Standpunkt und verliert dabei auf widersprüchliche Weise ihren theoretischen Primat. Dagegen bewahrt Apel die Asymmetrie zwischen dem transzendentalen Standpunkt und seinem Gegenstandsbereich, so gelingt es ihm gleichzeitig, die Perspektive der kritischen Diskurstheorie als ein bestimmtes Erkenntnisinteresse zu relativieren, das sich normativ von anderen praktisch begrenzten Erkenntnisinteressen unterscheidet. Es ist eben die transzendentale Selbstreflexion der praktischen Vernunft, die sich in praktisch eingeordnete Standpunkte aufspaltet, und kraft dieser synthetischen Artikulation der Reflexivität und Perspektivität mittels der Normativitätskategorie kann sich die Diskurstheorie als Standpunkt innerhalb ihres Gegenstandsbereichs einbeziehen, ohne ihre transzendentaltheoretische Asymmetrie und ihren praktischen Primat als Vernunftinteresse zur reflexiven Emanzipation zu verlieren.

\footnotetext{
${ }^{21}$ Jürgen Habermas, Erkenntnis und Interesse, Frankfurt 1968, S.240-246.

${ }^{22}$ Karl-Otto Apel, Transformation der Philosophie, Frankfurt am Main 1973, Bd.2, S.146.
} 